\title{
Apuntes para un abordaje multidimensional de las políticas públicas. Los puntos ciegos de la agenda feminista sobre las transferencias condicionadas de ingresos
}

\author{
DRA. NORA GOREN ${ }^{*}$
}

\section{Resumen:}

Este trabajo tiene como objetivo proponer una matriz multidimensional de abordaje analítica así cómo dejar formulados una serie de interrogantes que nos permitan avanzar en la construcción de la agenda feminista sobre los programas de Transferencia Condicionada de Ingresos.

Las esferas que consideramos son: la esfera estatal, el trabajo remunerado de las mujeres y las relaciones de reproducción en el hogar; a las cuales les incorporamos la agencia femenina, con la que buscamos hacer eje y darles protagonismo a las mujeres receptoras de los beneficios estatales. Así, a partir de un análisis dinámico de las relaciones sociales, nos proponemos que la categoría estatal de beneficiarias y/o sujetos de derechos le dé paso a la categoría de actoras sociales, que en lugar de reificarlas, las resitúe en el momento de la acción.

Palabras claves: Políticas públicas, agenda feminista, propuesta analítica

\section{Presentación}

os programas de Transferencias Condicionadas de Ingresos - TCl- constituyen una de las formas prioritarias que los Estados de la región han propuesto para combatir la pobreza. Si bien dichos programas no tienen como objetivo explícito moldear las relaciones de género, de hecho actúan sobre ellas.

En la literatura sobre la temática encontramos un fuerte énfasis en señalar la función maternalizadora y rematernalizadora de estas acciones. Si bien es cierto que éstos son los supuestos sobre los que estas políticas están diseñadas, no menos cierto es que estas miradas dejan fuera un abordaje multidimensional de las inequidades (Martinez Franzioni, 2012).

Goren, Nora "Apuntes para un abordaje multidimensional de las políticas públicas. Los puntos ciegos de la agenda feminista sobre las transferencias condicionadas de ingresos", en Zona Franca. Revista del Centro de Estudios Interdisciplinario sobre Mujeres, Año XXII, No 23, 2014, pp. 21-27.

Recibido: 12 de agosto 2014 - Aceptado: 2 de octubre 2014 
De este modo, y sobre la base de resultados de investigaciones que venimos llevando adelante en la Argentina sobre estos programas (Goren 2003, 2008, 2011, 2012, 2013) a partir de una perspectiva que sitúa en el centro del análisis a las relaciones sociales -más específicamente en las prácticas sociales, tal como señala Hirata Elena (1997)- buscamos reintroducir un análisis dinámico de la estructura social. Para ello nos planteamos como objetivos, por un lado, proponer una matriz multidimensional de abordaje analítico y por el otro, dejar formulados una serie de interrogantes.

En esta dirección retomamos el aporte de Sylvia Walby (1990), quien propone un abordaje de las relaciones de género a partir de seis estructuras: las relaciones de producción en el hogar; el trabajo remunerado; el Estado patriarcal; la violencia masculina; las relaciones patriarcales en el ámbito sexual; y las instituciones culturales patriarcales. En este sentido, y de acuerdo con los objetivos propuestos, consideraremos tres de las esferas reproductoras del orden patriarcal en tanto estructurales y estructurantes de las relaciones de género, y que nos aproximan a una mirada dinámica de las relaciones sociales.

Cada una de estas esferas y las dimensiones consideradas para su análisis nos brindan una aproximación a los supuestos y relaciones en que estos programas se inscriben y los que promueven. Al ponerlos en diálogo, lo que se busca es aproximarse a la dinámica de las relaciones sociales, que es justamente donde deseamos situar nuestro foco.

Las esferas que consideramos son: la esfera estatal, el trabajo remunerado de las mu- jeres y las relaciones de reproducción en el hogar; a las cuales les incorporamos la agencia femenina, con la que buscamos hacer eje y dar protagonismo a las mujeres receptoras del beneficio económico. Así nos proponemos que la categoría estatal de beneficiarias y/o sujetos de derechos dé paso a la categoría de actoras sociales, que en lugar de reificarlas, las resitúa en el momento de la acción.

El eje está puesto en la modalidad en los que los contenidos presentes en los diseños de las políticas son puestos en acto, en cuanto a las relaciones que las mujeres establecen en cada una de las esferas y en cómo éstas entran, a su vez, en relación. Así, el análisis conjunto de las relaciones que las mujeres establecen en sus unidades residenciales y las que establecen en y con el trabajo remunerado a partir de la percepción del beneficio otorgado por el Estado, busca significar y resignificar los supuestos y contenidos que este último presenta en su formulación. En definitiva, el foco se sitúa en el diálogo que se establece entre las esferas a partir de las relaciones presentes en cada una de ellas.

Tal como señala Giddens (1995), el momento de producción de la acción también es un momento de reproducción de los contextos en los que se lleva a cabo la vida social cotidiana. Al reproducir las propiedades estructurales, los agentes sociales también reproducen las condiciones que hacen esa acción posible. Las estructuras entonces no son independientes del conocimiento que tienen los actores sociales acerca de lo que hacen en su actividad cotidiana. Este conocimiento no es casual, sino "integral a la persistente estructuración de la vida social" (1995: 48).
Para poder ir construyendo la propuesta presentada y aproximarnos a un abordaje multidimensional de las formulaciones reproductoras del orden de género y colocar una serie de interrogantes, se dialogará en primer término con el lugar que la "pobreza" ha ocupado en los supuestos de la época y en la forma que ha moldeado a las políticas; luego nos centraremos en la letra escrita de los programas de transferencias condicionadas de ingresos y más específicamente en sus condicionalidades, y por último, en la dinámica del mercado de trabajo y el trabajo remunerado de las mujeres.

\section{Políticas para combatir la pobreza}

Las mujeres se fueron situando en el centro de las políticas de $\mathrm{TCl}$, en el marco de un fuerte desplazamiento que sufrieron los estudios de "la cuestión social", que implicó dejar de lado el análisis global del sistema en términos de explotación y reparto- para constituir un enfoque centrado en el segmento más vulnerable de la población, lugar en el que se las situó.

En este viraje, se comenzó a hablar de la exclusión como si fuera un estado dado y no como el resultado de un proceso, lo cual implicaba la intención de reducirla sin modificar las reglas del juego para todos/as (Rosanvallon; Fitousi, 1997:27). Asimismo, en estos estudios se señaló que las mujeres constituían "las más pobres de los pobres" y que la pobreza responde a las características de los hogares. En ellos se contempla más el estado conyugal de quienes los encabezan que los contextos sociales, políticos y económicos en los que se encuentran. Esto no sólo convierte a las mujeres 
en chivos expiatorios, sino que además desvía la atención de las grandes estructuras de desigualdad socioeconómica y de género (Moore, 2009). Implica también que la maternidad sólo es viable y/o aceptable en el contexto del matrimonio o bajo la égida de una jefatura de hogar masculina (Chant, 2006). La caracterización constante de las desventajas económicas de las unidades familiares con jefatura femenina que, implícita o explícitamente, son atribuidas a las circunstancias particulares de esos hogares, no sólo dan una imagen sesgada y devaluada de los enormes esfuerzos realizados por las mujeres jefas de hogar para superar los problemas que enfrentan por razones de género. Además borran el significado que tiene esa jefatura femenina del hogar para las mujeres (Chant; Sweetman, 2012).

En estas situaciones, en el marco de las relaciones de reproducción familiar, la maternidad se convierte en una fuente de poder, a través de la cual se accede a los beneficios brindados por el Estado. En estos espacios en que se van construyendo las "identidades asistidas" las mujeres se van apropiando de los recursos estatales, y se convierten así en expertas en su gestión. Ello en muchos casos las desplaza de la esfera privada cómo único y principal espacio de actuación. Se motorizan otras relaciones que los nuevos espacios implican, que se transforman entonces en potentes herramientas de acción, sin que eso suponga la eliminación de limitaciones y demarcaciones que la política pública contiene (Goren, 2008).

En estos contextos, los programas de Transferencias Condicionadas de Ingresos buscan, por un lado, aliviar la pobreza a corto plazo y, por otro, apoyar inversiones en capital humano que permitan el crecimiento económico a largo plazo (Sara Bradshaw, 2008). En América Latina tienen dos aspectos en común: por un lado, transferir ingresos monetarios, lo que puede estar acompañado por otro tipo de intervenciones, como son los programas de capacitación en oficios, actividades de sensibilización en temas sociales tales como salud sexual y reproductiva, promoción de actividades productivas, organización de actividades de participación comunitaria, etc.; y por el otro, establecen condicionalidades para la recepción del beneficio, cuya forma más habitual es la asistencia escolar y la atención sanitaria de los hijos e hijas (Rodríguez Enríquez, 2011).

En este sentido, algunas autoras (Molyneux, 2006; Chant, 2010) señalan que en estos programas las mujeres han sido depositarias de responsabilidades y obligaciones por sobre los derechos. Al decir de Lister (2006), las concepciones de ciudadanía fueron entrelazadas con dimensiones morales que regulan sus comportamientos, y han eclipsado el lugar de las mujeres y sus derechos detrás del bienestar futuro de sus hijos.

Es en este punto que nos preguntamos ¿cuáles son las dimensiones que están presentes en la naturalización de la relación de la madre con sus hijos/as?; ¿de qué manera estos dispositivos de control son apropiados, transformados y reconocidos por las mujeres en sus prácticas cotidianas?; ¿cuáles serían los interrogantes que nos permitirían encontrar los espacios para abrir brechas y/o rupturas?

En esta dirección, nos surgen aún otros interrogantes: ¿podemos pensar al Estado como el único actor que reproduce la lógica patriarcal? ¿No sucede lo mismo con las relaciones sociales sobre las que el Estado actúa? ¿No son éstas también reproductoras y productoras del orden patriarcal?

Las mujeres, en general, se reconocen e identifican como las encargadas y responsables del cuidado de sus hijos/as, roles y espacios que rara vez quieren ceder a sus convivientes: "No dejaría que mi marido vaya a la reunión de padres, se va a quedar con la boca cerrada"; "si dejo a mi marido que lleve a mi hijo al médico no va a saber explicarle que es lo que ellos tienen".

Un modo de comprender estas expresiones es enmarcándolas en la estructura social y en las representaciones que ellas sostienen en tanto "sujetos sociales totales". En este punto, la dimensión del cuidado se halla fuertemente anclada a modo de disposición y reconocimiento subjetivo, y, más aún, como forma de pertenencia a una comunidad. Campo, que por cierto, también está atravesado por el lugar, significado y prácticas que han asumido los discursos de derechos y la legislación, dando paso a un entramado de posibilidades y de agenciamiento mucho más complejo.

\section{Letra escrita de los programas}

En la letra escrita del programa y considerando la Asignación Universal por Hijo implementada en la Argentina a partir del año 2009 , se observa que en la misma se señala que "los requisitos que deberán acreditarse... están condicionados al cumplimiento de los controles sanitarios obligatorios para menores y a la concurrencia al sistema público de enseñanza".

En la misma dirección hubo un interés explícito, expresado 
en el artículo 10 de la Resolución 393 de la ANSES, y expresado por un funcionario de dicho organismo de la siguiente manera: "se instruyó para que fueran las madres quienes recibieran la asignación, ya que ellas lo gastarán en lo necesario". $\mathrm{O}$, en palabras de las receptoras del beneficio, "cuando venían a difundir el programa, nos decían que teníamos que ser nosotras las que nos inscribiéramos", "la asistente social les explicaba a las mamás que ellas son más responsables que los papás para cuidar a sus hijos", "si las mamás somos más responsables, siempre recae sobre nosotras, aunque yo creo que debería cambiar y deberíamos compartirlo".

Así, desde la letra escrita del programa no se problematiza el lugar de las mujeres en las relaciones familiares. Ello entra en tensión con acuerdos nacionales e internacionales suscriptos por la Argentina, en los cuales el país se compromete a promover la igualdad y la equidad entre varones y mujeres. Lo cual supondría la implementación de acciones de promoción de responsabilidades familiares compartidas, en las que Estado debería jugar un papel central.

No obstante, si bien Estado es patriarcal, tal como lo señala Brown (1992), eso no significa que sea monolítico, ni en cuanto a sus acciones y la forma que éstas adoptan, ni en cuanto a la modalidad en que circulan sus discursos al interior de las instituciones; ni tampoco respecto de la forma en que éstos se plasman en acciones de política pública. De igual modo, las mujeres receptoras de estas acciones están también atravesadas por múltiples acciones estatales, nacionales, provinciales y locales, que no siempre van en la misma dirección, sino que más bien entran en tensión.

En términos de género, la mujer sigue siendo la principal responsable del cuidado y/o de la gestión de éste. La evidencia empírica muestra, tal como lo señala Eleonor Faur (2012), que estas responsabilidades atraviesan a todos los sectores sociales, aunque con especificidades de acuerdo con cada uno de ellos.

Ahora bien, pensándolo de manera contextualizada y haciendo una reflexión respecto de potenciales cambios al interior del programa, nos preguntamos ¿qué sucedería si el beneficio estuviera destinado de manera indistinta a varones y mujeres?, ¿la percepción de un monto de dinero por parte de los varones implicaría un cambio en términos de relaciones familiares, 0 se estaría reafirmando el modelo patriarcal? Todo hace suponer que van a seguir siendo las mujeres las que lleven adelante estas actividades, pero en este caso, sin retribución económica y con la pérdida de las afirmaciones subjetivas que la percepción de un ingreso monetario implica en sus vidas, lo cual obtura así la potencialidad de cambio que esto implica.

\section{Trabajo reproductivo remunerado}

El trabajo remunerado de las mujeres y lo que figura en la letra escrita de la AUH son dos elementos que parecieran entrar en tensión, entonces nos preguntamos ¿cuál es el entramado que subyace a esta relación y cómo abordarla?

Por un lado, una de las incompatibilidades de la $\mathrm{AUH}$, expresada en el decreto que le da lugar, determina que la asignación es incompatible con el cobro de prestaciones contributivas o no contributivas de orden nacional, provincial, municipal, o de la Ciudad Autónoma de Buenos Aires. Ello implica que las mujeres, entonces, principales receptoras de este beneficio, quedan excluidas de otras prestaciones, como lo son otros programas que se promueven desde el Estado Nacional en búsqueda de mejorar las condiciones de empleabilidad de la población, tal como el Seguro de Capacitación y Empleo y el programa Jóvenes por Más y Mejor Trabajo. Su acceso al mercado de trabajo en mejores condiciones, y la posibilidad de progresar en relación con sus competencias laborales quedarían, entonces, restringidos. No obstante, esta aseveración es relativizada a la luz de lo señalado por un funcionario del Ministerio de Trabajo (entrevistado en octubre de 2012), quien expresa que las mujeres que han transitado por el Seguro de Capacitación y Empleo no han mejorado su inserción en el mercado laboral, como sí sucede en el caso de los varones. Teniendo en cuenta la limitación en la concepción de uno de los requisitos, creemos importante detenernos en el tipo de inserción efectiva que las mujeres tienen en el "mercado de trabajo", y específicamente de aquellas que perciben la $\mathrm{AUH}$, para plantearnos que, de no existir esa incompatibilidad, su situación mejoraría.

Por un lado, es de destacar que una gran proporción de las mujeres receptoras de estos beneficios nunca ha participado en el "mercado de trabajo". A pesar de la llamada feminización de la mano de obra operada en las últimas décadas, su participación es sustancialmente menor, dado que representan, en general, menos de la mitad de quienes 
reciben una remuneración a cambio de un trabajo.

En los casos de las que participan del "mercado de trabajo", sus inserciones se caracterizan por ser de bajos ingresos y/o por estar ubicadas en la economía informal. En el marco de un mercado fuertemente segmentado, las actividades vinculadas al cuidado y la entrega al otro/a, se enmarcan en las de menor valorización y posibilidades de desarrollo personal y profesional. Esto nos permite señalar que las oportunidades que ofrece el mercado de trabajo para las mujeres receptoras de estos beneficios son escasas. En segundo lugar, que si acceden a un empleo lo hacen en ciertos segmentos caracterizados por la informalidad y los más bajos ingresos. Por otra parte, la presencia de niños/as en los hogares condiciona la participación laboral de estas mujeres, y más aún en los trabajos registrados.

Entonces, esa incompatibilidad ¿afecta realmente la posibilidad de inserción laboral de las mujeres, en un mercado caracterizado por la segmentación laboral, en el que los territorios femeninos y masculinos están fuertemente consolidados? ¿No sería adecuado pensar cómo incidir en los determinantes de los procesos de demanda laboral y en realizar cambios en la organización de los tiempos de trabajo y en los espacios de cuidado infantil y de servicios de cuidado para adultos, de manera articulada y conjunta?

La corresponsabilidad social requiere, por un lado, sumar a los varones y, por el otro, incluir a otros actores sociales. De lo contrario, podría ocurrir que desde la protección social se demandara la participación de los varones, mientras que desde el mercado laboral ésta les fuera impedida. Esto nos lleva a pen- sar en acciones tales como un sistema de licencia más amplio, hasta otro tipo de organización de las relaciones laborales que contemple tanto la demanda como la organización de los tiempos de trabajo en función de familias que requieren de atención y cuidados tanto para mujeres como para varones.

Ahora bien, resulta instigador formular una serie de interrogantes que nos permitan empezar a pensar en otro tipo de organización de lo social y de los tiempos destinados a cada una de las esferas que la componen, no ya como espacios separados, sino en íntima interrelación. ¿Por qué los horarios escolares raramente coinciden con los laborales? ¿Por qué las licencias vinculadas a temas de cuidado están principalmente destinadas a las mujeres? ¿Por qué los horarios de reuniones y actividades laborales se enciman con los horarios que se destinan al estar con los afectos? ¿Por qué los salarios de las actividades realizadas por las mujeres son inferiores? ¿Sobre qué modelo familiar se basa la organización de la sociedad?

La actual organización de los tiempos, tanto los prescriptos por los programas como los sociales, parecerían considerar el modelo tradicional de la mujer ama de casa a tiempo completo, que realiza todas las tareas de cuidados necesarios. $Y$ si esta mujer quiere incorporarse al mercado laboral, en general se piensa que es su responsabilidad individual resolver previamente la organización familiar. Es decir, las organizaciones e instituciones sociales $-y$ la sociedad en general- siguen sin considerar al cuidado de la vida humana como una responsabilidad social y política. Esta percepción asume mayor intensidad en el colectivo de mujeres perceptoras de estos programas. Aquellas que en algún momento llevaron adelante, por ejemplo, alguna actividad mercantil, señalan, por ejemplo, que "el sueldo se nos iba en guardería", como si el espacio de cuidado infantil lo tuviera que pagar la mujer; y por el otro, con las limitaciones de participar en trabajos que podrían, o más bien "deberían", constituirse en un espacio de crecimiento personal y profesional, más allá del mero hecho de generar un ingreso monetario.

Así, a la vez que las mujeres se han ido integrando al mercado laboral, ha ido desapareciendo el modelo familiar "varón proveedor de ingresos - mujer ama de casa" y ha comenzado a abrirse paso un nuevo modelo que tiende a consolidarse. El varón mantiene su rol casi intacto, pero la figura del ama de casa tradicional tiende a desaparecer, lo cual no significa que la mujer abandone sus tareas de cuidadora y gestora del hogar, sino que, de hecho, asume un doble o triple papel: el familiar y el laboral y, de más está señalar, el comunitario.

Este proceso de incorporación laboral de las mujeres les ha significado introducirse en un mundo definido y construido por y para los varones. Un mundo -el mercantil- que sólo puede funcionar de la manera en que lo hace porque se apoya y depende del trabajo familiar. Un mundo para el que se requiere libertad de tiempos y espacios; es decir, que exige la presencia de alguien en la casa que realice las actividades básicas para la vida. En este sentido, las mujeres receptoras de estos programas, ¿a qué trabajos podrán acceder en el marco de esta organización laboral? El modelo masculino de participación laboral no es generalizable. Si 
las mujeres lo imitaran, ¿quién cuidaría de la vida humana, con toda la dedicación que ello implica? ¿Acaso este modelo no es el que subyace a que los empleadores prefieran varones? ¿No es este modelo el que hace que los trabajos que implican menor posibilidad de crecimiento y de trayectoria sean realizados por mujeres?

\section{Reflexiones finales}

A lo largo de este artículo, buscamos aportar a un abordaje multidimensional de las políticas públicas, desde una perspectiva dinámica de las relaciones sociales. Eso nos llevó a proponer tres esferas reproductoras del orden patriarcal: el Estado, el trabajo reproductivo y el productivo de las mujeres, y ponerlas en dialogo en relación con el concepto de agencia.
Asimismo, hemos propuesto una serie de interrogantes, en muchos casos contrafácticos en tanto disparadores para pensar formas y posibilidades de políticas que conduzcan a un camino deconstructor de los regímenes de género. Se reflexiona sobre los espacios reproductores del orden de género, en donde si bien el Estado ocupa un lugar central, también lo hacen las relaciones sociales sobre las que éste actúa.

Así, las prácticas de las mujeres no se condicen con los mandatos discursivos en los que los distintos "programas" se inscriben, y responden también a una experiencia acumulada, en la que confluyen necesidades, discursos y prácticas políticas.

De esta manera en el diálogo/tensión entre la estructura y la práctica, se ponen en acto juegos de poder y de resistencia que marcan la relación entre la subjetividad y la estructura social. Este conjunto de factores permiten dar cuenta de la manera en que, dentro de las estructuras de subordinación en que están inmersas las mujeres, éstas resisten el orden dominante masculino subvirtiendo los significados hegemónicos y reutilizándolos para sus propios intereses y agendas.

Por último, difícil sería suponer que los programas sociales en general y los de TCl en particular puedan cambiar la estructura económica o los elementos que subyacen a la estructura de la economía que producen y reproducen la pobreza, ni pueden cambiar por sí mismos las pautas de funcionamiento del orden patriarcal. Pero sí tienen la potencialidad de aportar a los procesos de transformación allí donde las desigualdades permanecen.

Notas:

* Dra por la Universidad de Buenos Aires con mención en Sociología, DocenteInvestigadora Universidad Nacional Arturo Jauretche -UNAJ-;Universidad Nacional de Buenos Aires; norgoren@gmail.com

\section{Bibliografía:}

BRADSHAW, Sarah (2008) From structural adjustment to social adjustment: a gendered analysis of conditional cash transfer programmes in Mexico and Nicaragua. Global Social Policy, V. 8, n 2, p. 188-207

BROWN, Wendy (1992) Finding the man in the state. Feminist Studies, Vol 18, N:1 CARRASCO, Cristina (2003) La sostenibilidad de la vida humana ¿Un asunto de mujeres? En: Mujeres y trabajo: cambios impostergables. Porto Alegre: Veraz Comunicação.

CHANT, Sylvia (2006). Not incomes but inputs: critiquing the "feminisation of poverty" and the "feminisation" of anti-poverty programmes. Paper presentado en International Seminar Development and Vulnerability.

CHANT, Sylvia.; SWEETMAN, C.(2012) Fixing women or fixing the world? 'Smart economics', efficiency approaches, and gender equality in development, Gender \& Development, v 20, n3, p. 517-529. 
DUBET, Francois (2011). Repensar la justicia social. Contra el mito de la igualdad de oportunidades. Buenos Aires: Siglo XXI.

FAUR, Eleonor 2012 "El cuidado infantil desde la perspectiva de las mujeres-madres. Un estudio en dos barrios populares del Área Metropolitana de Buenos Aires", en V. Esquivel, E. Faur, E. Jelin, F. Pereyra y M. Bruno (eds.), Las lógicas del trabajo infantil, pp. 107-160. IDES, Buenos Aires.

FRASSER, Nancy (1996) Reconsiderando la esfera pública: una contribución a la crítica de la democracia realmente existente. Entrepasados, Revista de Historia, n. 7.

GIDDENS, Anthony. (1995) La constitución de la sociedad. Bases para la teoría de la estructuración. Madrid: Amorrortu.

GOREN, Nora (2003) Políticas sociales, identidades, pobreza y género. CONGRESO NACIONAL DE ESTUDIOS DEL TRABAJO, 6. Buenos Aires.

(2011) Entre la autonomía y la dependencia. Interpelando las políticas de empleo desde una perspectiva de género. Sociologías V.13, p. 318-341.

(2013) Una relación conflictiva. Trabajo no remunerado vs trabajo remunerado en los programas de transferencia condicionada de ingresos. Aljaba.

(2013) Repensando el trabajo de las mujeres en los programas de transferencia condicionada de ingresos en Trabalhadoras - Análise da Feminização das Profissões e Ocupações / Yannoulas, Silvia Cristina (Coord.) Editorial Abaré.

HIRATA, Helena. y KERGOAT, Daniele. (1997) La división sexual del trabajo. Permanencia y cambio. Asociación Trabajo y Sociedad. Centro de Estudios de la Mujer. CEIL/PIETTE. Buenos Aires.

MARTíNEZ Franzoni, Juliana (2012), Blacks, Whites, or Grays? Conditional Transffers and Gender Equality in Latin America. Social Politics 2012, Vol:0

MOLYNEUX, Maxine (2002) Gender and the silences of social capital: lessons from Latin America. Development and Change, n. 33, 2002.

LISTER, Ruth (2006): Children (but not women) first: New Labour, child welfare and gender. En Critical Social Policy, Vol. 26 (2): 294-314.

MOORE, Ch. (2009) Nicaragua's red de protección social. Country Study 17. Brasilia: International Policy Center (IPC)

ROSANVALLON, Pierre; FITOUSSI, Jean Paul (1997). La nueva era de las desigualdades. Buenos Aires: Manatial.

RODRÍGUEZ ENRÍQUEZ, Corina (2011) Programas de transferencias condicionadas de ingreso e igualdad de género ¿Por dónde anda América Latina? Serie Mujer y Desarrollo, n. 109. Santiago de Chile: División de Asuntos de Género. 